\title{
X-ray photon diagnostics at the European XFEL
}

\section{Jan Grünert, Marc Planas Carbonell, Florian Dietrich, Torben Falk, Wolfgang Freund, Andreas Koch, Naresh Kujala, Joakim Laksman, Jia Liu, Theophilos Maltezopoulos, Kai Tiedtke, Ulf Fini Jastrow, Andrey Sorokin, Evgeny Syresin, Alexander Grebentsov and Oleg Brovko}

J. Synchrotron Rad. (2019). 26, 1422-1431

\section{IUCr Journals CRYSTALLOGRAPHY JOURNALS ONLINE}

Copyright (C) International Union of Crystallography

Author(s) of this article may load this reprint on their own web site or institutional repository provided that this cover page is retained. Republication of this article or its storage in electronic databases other than as specified above is not permitted without prior permission in writing from the IUCr.

For further information see http://journals.iucr.org/services/authorrights.html 


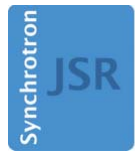

JOURNAL OF SYNCHROTRON RADIATION

ISSN 1600-5775

Received 16 October 2018

Accepted 8 May 2019

Edited by P. Fuoss, SLAC National Accelerator Laboratory, USA

${ }^{1}$ This article will form part of a virtual special issue on $\mathrm{X}$-ray free-electron lasers.

Keywords: XFEL physics; free-electron lasers; photon diagnostics; hard X-rays; soft X-rays; instrumentation.

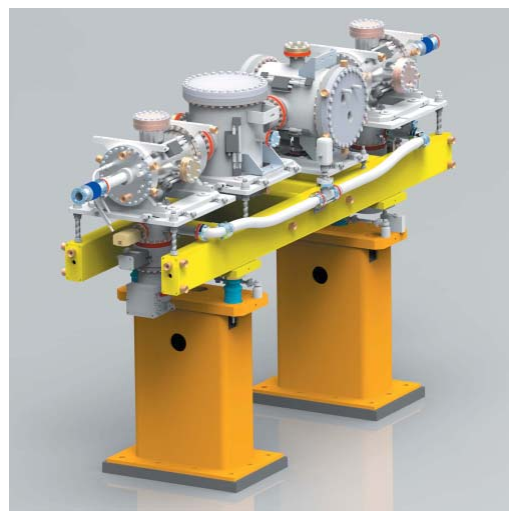

(C) 2019 International Union of Crystallography

\section{X-ray photon diagnostics at the European $\mathrm{XFEL}^{1}$}

\author{
Jan Grünert, ${ }^{\mathrm{a} *}$ Marc Planas Carbonell, ${ }^{\mathrm{a}}$ Florian Dietrich, ${ }^{\mathrm{a}}$ Torben Falk, \\ Wolfgang Freund, ${ }^{a}$ Andreas Koch, ${ }^{a}$ Naresh Kujala, ${ }^{a}$ Joakim Laksman, ${ }^{a}$ Jia Liu, \\ Theophilos Maltezopoulos, ${ }^{a}$ Kai Tiedtke, ${ }^{\text {b }}$ Ulf Fini Jastrow, ${ }^{\text {b }}$ Andrey Sorokin, ${ }^{b}$ \\ Evgeny Syresin, ${ }^{\mathrm{c}}$ Alexander Grebentsov ${ }^{\mathrm{c}}$ and Oleg Brovko ${ }^{\mathrm{c}}$
}

${ }^{\mathbf{a}}$ European XFEL, Holzkoppel 4, Schenefeld 22869, Germany, ${ }^{\mathbf{b}}$ Deutsches Elektronen-Synchrotron (DESY), Notkestraße 85, Hamburg 22603, Germany, and 'Joint Institute for Nuclear Research (JINR), Joliot-Curie Straße 6, Dubna, Moscow Region 141980, Russian Federation. *Correspondence e-mail: jan.gruenert@xfel.eu

The European X-ray Free-Electron Laser (European XFEL) (Altarelli et al., 2006; Tschentscher et al., 2017), the world's largest and brightest X-ray freeelectron laser (Saldin et al., 1999; Pellegrini et al., 2016), went into operation in 2017. This article describes the as-built realization of photon diagnostics for this facility, the diagnostics commissioning and their application for commissioning of the facility, and results from the first year of operation, focusing on the SASE1 beamline, which was the first to be commissioned. The commissioning consisted of pre-beam checkout, first light from the bending magnets, X-rays from single undulator segments, SASE tuning with many undulator segments, first lasing, optics alignment for FEL beam transport through the tunnel up to the experiment hutches, and finally beam delivery to first users. The beam properties assessed by photon diagnostics throughout these phases included per-pulse intensity, beam position, shape, lateral dimensions and spectral properties. During this time period, the machine provided users with up to $14 \mathrm{keV}$ photon energy, $1.5 \mathrm{~mJ}$ pulse energy, $300 \mathrm{FEL}$ pulses per train and $4.5 \mathrm{MHz}$ intra-bunch train repetition rate at a $10 \mathrm{~Hz}$ train repetition rate. Finally, an outlook is given into the diagnostic prospects for the future.

\section{Introduction}

The European XFEL is a linear-accelerator based light source that generates X-rays a billion times brighter than conventional synchrotron X-ray sources. It is designed to deliver up to $27000 \mathrm{X}$-ray pulses per second thanks to its superconducting linear accelerator. The facility started photon commissioning in April 2017 and went into operation on 1 July 2017. First lasing at hard X-ray energies was observed in May 2017 using the SASE1 undulator, and first lasing with soft $\mathrm{X}$-rays was achieved with the SASE3 undulator in February 2018. User experiments have been conducted since September 2018 on the SASE1 beamline at the science instruments Femtosecond X-ray Experiments (FXE; Galler et al., 2019) and Single Particles, Clusters, and Biomolecules and Serial Femtosecond Crystallography (SPB/SFX; Mancuso et al., 2019). In November 2018, two more instruments were taken into operation, and the last two baseline instruments went online in spring 2019. Here, we globally describe the European XFEL only as far as to outline the special requirements imposed on photon diagnostics and to illustrate how the photon diagnostics instrumentation is embedded in the overall facility. An overview of the facility with descriptions of the accelerator, experiments and photon system is given by Tschentscher et al. (2017).

$\mathrm{X}$-ray FEL radiation is characterized by its ultrashort pulse duration, high pulse energies and high degree of transverse 
coherence. Additional outstanding features of the European XFEL are the high repetition rate of up to $4.5 \mathrm{MHz}$ within a pulse train and the high electron beam energy of up to $17.5 \mathrm{GeV}$, which provides fundamental lasing up to $24 \mathrm{keV}$. On the one hand, any photon diagnostics had to be built to survive single-shot and multi-bunch damage; on the other hand, it should provide maximum information about the X-ray beam, preferably with shot-to-shot resolution and with the least interference to the beam delivery and experiments. The time between successive pulses can be as short as $220 \mathrm{~ns}$, which defines the maximum measurement duration for pulseresolved detection. The design plans (Grünert, 2012; Grünert et al., 2012, 2013, 2015) took all of this into account, detailing methods and special instrumentation for the commissioning phase as well as the operation phase diagnostics.

Monitoring the photon pulses generated by the European XFEL is mandatory for startup and operation. The photon diagnostic systems provide essential information to the machine operators for setup, operation and optimization of the accelerator, undulators and X-ray optics during every phase, starting from beam commissioning throughout user operation. Diagnostics are also mandatory for normalization and interpretation of the experimental data by the users. Several beam properties are measured online for each photon pulse and with minimal distortion. The pulse energy, beam position, spectral content and temporal properties can be collected using these systems. Pulse-to-pulse capability is challenging because of the high repetition rate, which, at up to
4.5 MHz, is several orders of magnitude higher than at other existing hard X-ray FEL facilities. However, it is important to be able to normalize data for fluctuations of photon pulse parameters due to the SASE process or due to electron or X-ray beam instabilities. In addition, for setup and specific measurements, several invasive photon diagnostic systems are installed which stop the X-ray pulses or severely modify the pulse properties.

Here we focus on photon diagnostic devices installed in the photon transport sections inside the tunnels, particularly in the hard X-ray SASE1 beamline. Some initial SASE1 commissioning results from measurements in 2017 were reported by Grünert et al. (2018), showing basic spectroscopy with $K$-edge absorption in metal filters and the first SASE1 intensity measurements. In the current paper, a comprehensive set of commissioning results, mainly for the SASE1 diagnostics, is presented for the beam conditions used throughout the 2018 user beam times. The sequence of tunnel diagnostics devices is shown schematically in Fig. 1. Their precise positions and names are listed for reference in Fig. 2 where the locations are quoted in accelerator coordinates with the origin at the electron gun. The distances in Fig. 1 are shown in photon system coordinates, which is a useful format for X-ray optics calculations and measures the distance from the FEL source point in the middle of the third-last undulator segment. This source point location in each beamline is also listed in Fig. 2.

Further diagnostics are integrated into the scientific instrumentation in the experiment hall. This includes the

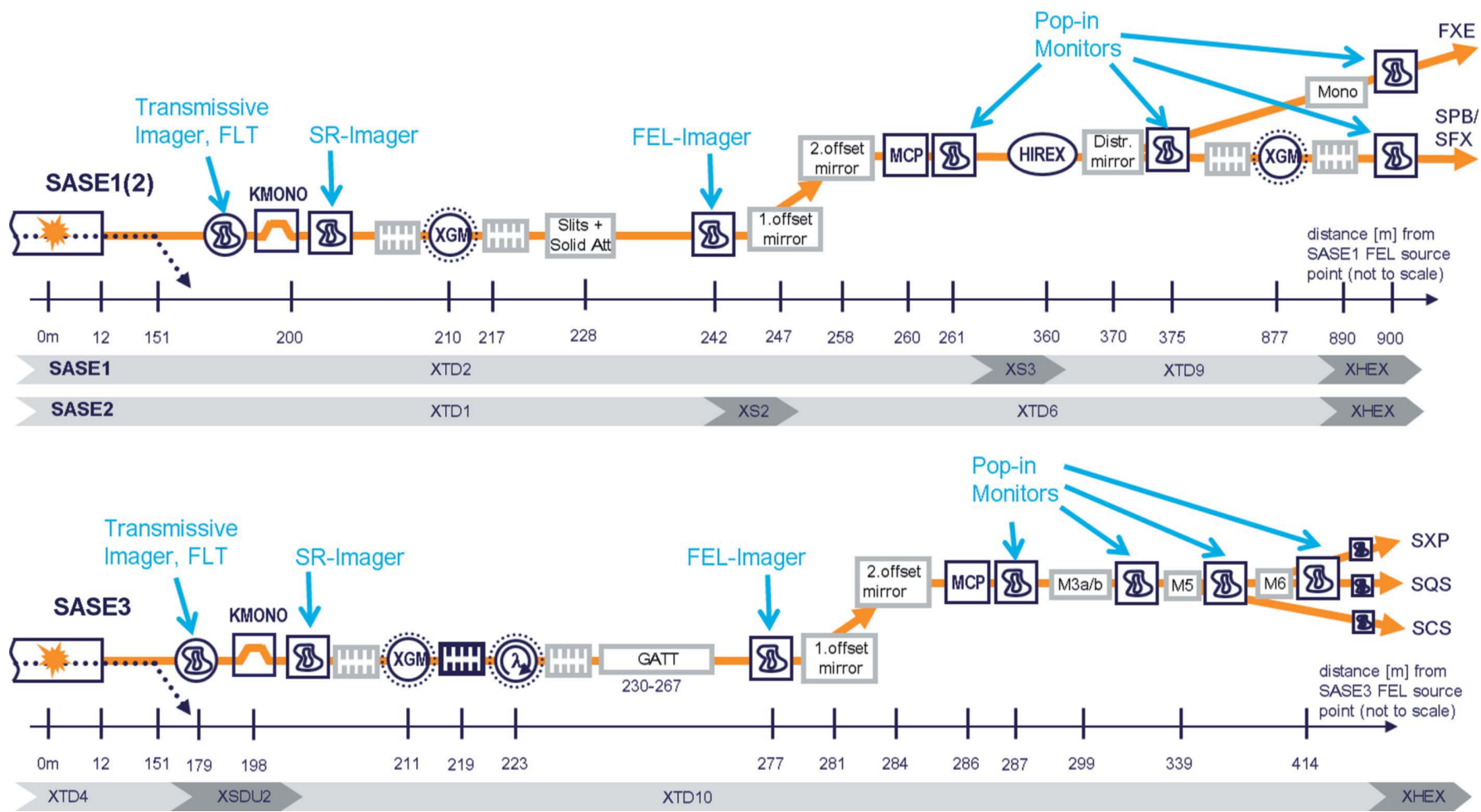

Figure 1

Schematic layout of the photon diagnostics (in black) in all three FEL beamlines - SASE1, SASE2 and SASE3 - including the relevant X-ray optics (in grey) and in the sequence in which they are positioned in the tunnels; see also the optical layout given by Tschentscher et al. (2017). Precise positions are listed in Fig. 2. Light blue arrows point to the imaging stations. The locations in SASE1 and SASE3 are shown in photon system coordinates as relative distances to the FEL source point. The names of the tunnels (XTDx) and shaft buildings (XSx) are indicated below the position axis. 


\begin{tabular}{|c|c|c|c|c|c|c|c|}
\hline 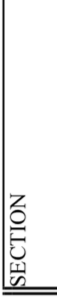 & 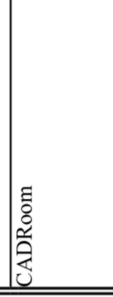 & 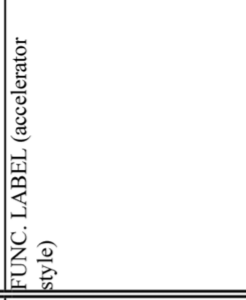 & 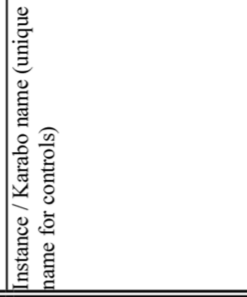 & 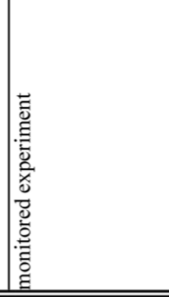 & 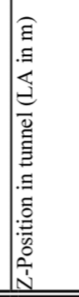 & 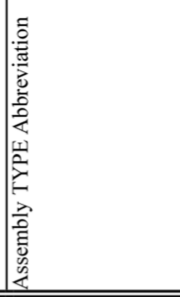 & 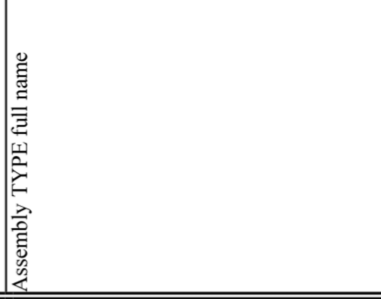 \\
\hline \multicolumn{8}{|c|}{ SASE1 } \\
\hline T9 & XTD2 & & & & 2238,6 & & Undulator start \\
\hline T9 & XTD2 & & & & 2433,8 & & Undulator Segment U33 (source point) \\
\hline T9 & XTD2 & & & & 2446,0 & & Undulator end \\
\hline T9 & XTD2 & FLT.2615.T9 & SA1_XTD2_FLT & SPB/SFX; FXE & 2615,1 & FLT & Filter Chamber \\
\hline T9 & XTD2 & IMGTR.2615.T9 & SAl_XTD2_IMGTR & SPB/SFX; FXE & 2615,1 & IMGTR (OTR-C) & Transmissive imager \\
\hline $\mathrm{T9}$ & XTD2 & KMONO.2634.T9 & SA1_XTD2_KMONO & SPB/SFX; FXE & 2634,0 & KMONO & K-Monochromator \\
\hline T9 & XTD2 & IMGSR.2634.T9 & SAl_XTD2_IMGSR & SPB/SFX; FXE & 2634,5 & IMGSR & SR Imager \\
\hline T9 & XTD2 & XGM.2643.T9 & SA1_XTD2_XGM & SPB/SFX; FXE & 2643,4 & $\mathrm{XGM}$ & X-ray Gas Monitor \\
\hline T9 & XTD2 & DP.2650.T9 & SA1_XTD2_VAC/... & SPB/SFX; FXE & 2650,4 & $\mathrm{DP}$ & Differential Pumping by WP74 \\
\hline T9 & XTD2 & PES.2654.T9 & SA1_XTD2_PES & SPB/SFX; FXE & 2653,7 & PES & PhotoElectron Spectrometer \\
\hline T9 & XTD2 & IMGFEL.2676.T9 & SA1_XTD2_IMGFEL & SPB/SFX; FXE & 2676,3 & IMGFEL & FEL Imager \\
\hline T9 & XTD2 & MCP.2693.T9 & SA1_XTD2_MCP & SPB/SFX; FXE & 2693,4 & MCP & MCP-based detector \\
\hline T9 & XTD2 & IMGPII45.2694.T9 & SA1_XTD2_IMGPII45 & SPB/SFX; FXE & 2694,4 & IMGPII45 & Pop-in Monitor Type II-45 \\
\hline T9 & XTD9 & HIREX.2794.T9 & SAl_XTD9_HIREX & SPB/SFX; FXE & 2794,1 & HIREX & High REsolution hard X-ray spectrometer \\
\hline T9 & XTD9 & IMGPII45.2809.T9.SPB & SPB XTD9 IMGPII45 & SPB & 2808,8 & IMGPII45 & Pop-in Monitor Type II-45 \\
\hline T9 & XTD9 & XGM.3311.T9.SPB & SPB XTD9 XGM & SPB & 3311,0 & XGM & X-ray Gas Monitor \\
\hline T9 & XTD9 & IMGPI.3334.T9.SPB & SPB XTD9 IMGPI & SPB & 3334,2 & IMGPI & Pop-in Monitor Type I \\
\hline T9 & XTD9 & IMGPI.3334.T9.FXE & FXE_XTD9_IMGPI & FXE & 3334,2 & IMGPI & Pop-in Monitor Type I \\
\hline \multicolumn{8}{|c|}{$\overline{\text { SASE2 }}$} \\
\hline$\overline{T 6}$ & XTD1 & & & & 2200,5 & & Undulator start \\
\hline T6 & XTD1 & & & & 2395,5 & & Undulator Segment U33 (source point) \\
\hline T6 & XTD1 & & & & 2407,7 & & Undulator end \\
\hline T6 & XTD1 & FLT.2576.T6 & SA2_XTD1_FLT & HED ; MID; N.C. & 2576,7 & FLT & Filter Chamber \\
\hline T6 & XTD1 & IMGTR.2576.T6 & SA2 XTD1_IMGTR & HED ; MID; N.C. & 2576,7 & IMGTR (OTR-C) & Transmissive imager \\
\hline T6 & XTD1 & KMONO.2586.T6 & SA2_XTD1_KMONO & HED ; MID; N.C. & 2586,2 & KMONO & K-Monochromator \\
\hline T6 & XTD1 & IMGSR.2586.T6 & SA2 XTD1 IMGSR & HED ; MID ; N.C. & 2586,8 & IMGSR & SR Imager \\
\hline T6 & XTD1 & XGM.2595.T6 & SA2 XTD1 XGM & HED ; MID ; N.C. & 2595,4 & XGM & X-ray Gas Monitor \\
\hline T6 & XTD1 & IMGFEL.2637.T6 & SA2_XTD1_IMGFEL & HED ; MID; N.C. & 2637,3 & IMGFEL & FEL Imager \\
\hline T6 & XTD6 & PES.2676.T6 & SA2 XTD6 PES & HED ; MID; N.C. & 2676,2 & PES & PhotoElectron Spectrometer \\
\hline T6 & XTD6 & MCP.2699.T6 & SA2 XTD6_MCP & HED ; MID; N.C. & 2698,6 & $\mathrm{MCP}$ & MCP-based detector \\
\hline T6 & XTD6 & IMGPII45-1.2700.T6 & SA2 XTD6 IMGPII45-1 & HED ; MID; N.C. & 2700,1 & IMGPII45 & Pop-in Monitor Type II-45 \\
\hline T6 & XTD6 & HIREX.2776.T6 & SA2 XTD6_HIREX & HED ; MID; N.C. & 2775,7 & HIREX & High REsolution hard X-ray spectrometer \\
\hline T6 & XTD6 & IMGPII45-2.2795.T6 & SA2_XTD6_IMGPII45-2 & HED ; MID; N.C. & 2795,2 & IMGPII45 & Pop-in Monitor Type II-45 \\
\hline T6 & XTD6 & & & N.C. SA2 & & IMGPII45 & Pop-in Monitor Type II-45 \\
\hline T6 & XTD6 & XGM.3261.T6.HED & HED_XTD6_XGM & HED & 3261,1 & XGM & X-ray Gas Monitor \\
\hline T6 & XTD6 & IMGPI.3333.T6.MID & MID_XTD6_IMGPI & MID & 3333,8 & IMGPI & Pop-in Monitor Type I \\
\hline T6 & XTD6 & IMGPI.3333.T6.HED & HED XTD6_IMGPI & HED & 3333,8 & IMGPI & Pop-in Monitor Type I \\
\hline T6 & XTD6 & & & N.C. SA2 & 3333,8 & IMGPI & Pop-in Monitor Type I \\
\hline \multicolumn{8}{|c|}{ SASE3 } \\
\hline 1710 & XTD4 & & & & 2803,5 & & Undulator start \\
\hline T10 & XTD4 & & & & 2919,4 & & Undulator Segment U20 (source point) \\
\hline T10 & XTD4 & & & & 2931,6 & & Undulator end \\
\hline T10 & XTD4 & FLT.3098.T10 & SA3_XSDU2_FLT & SQS; SCS;SQS2 & 3098,0 & FLT & Filter Chamber \\
\hline T10 & XTD4 & IMGTR.3099.T10 & SA3_XSDU2_IMGTR & SQS; SCS;SQS2 & 3099,0 & IMGTR (OTR-C) & Transmissive imager \\
\hline T10 & XTD10 & KMONO.3117.T10 & SA3 XTD10 KMONO & SQS; SCS;SQS2 & 3117,2 & KMONO & K-Monochromator \\
\hline T10 & XTD10 & IMGSR.3118.T10 & SA3 XTD10_IMGSR & $\mathrm{SQS} ; \mathrm{SCS} ; \mathrm{SQS} 2$ & 3118,0 & IMGSR & SR Imager \\
\hline$T 10$ & XTD10 & XGM.3130.T10 & SA3_XTD10_XGM & $\mathrm{SQS} ; \mathrm{SCS} ; \mathrm{SQS} 2$ & 3130,1 & XGM & X-ray Gas Monitor \\
\hline$T 10$ & XTD10 & DP.3138.T10 & SA3_XTD10_VAC/.. & SQS; SCS;SQS2 & 3138,4 & DP & Differential Pumping by WP74 \\
\hline$T 10$ & XTD10 & PES.3142.T10 & SA3_XTD10_PES & SQS; SCS;SQS2 & 3142,2 & PES & PhotoElectron Spectrometer \\
\hline$T 10$ & XTD10 & IMGFEL.3196.T10 & SA3_XTD10_IMGFEL & SQS; SCS;SQS2 & 3196,4 & IMGFEL & FEL Imager \\
\hline$T 10$ & XTD10 & MCP.3205.T10 & SA3 XTD10_MCP & SQS; SCS;SQS2 & 3205,1 & MCP & MCP-based detector \\
\hline$T 10$ & XTD10 & IMGPIII.3206.T10 & SA3_XTD10_IMGPIII & SQS; SCS;SQS2 & 3206,0 & IMGPIII & Pop-in Monitor Type III \\
\hline T10 & XTD10 & IMGPII90.3221.T10 & SA3 XTD10_IMGPII90 & $\mathrm{SQS} ; \mathrm{SCS} ; \mathrm{SQS} 2$ & 3221,2 & IMGPII90 & Pop-in Monitor Type II-90 \\
\hline T10 & XTD10 & IMGPII45.3260.T10.SQS & SA3_XTD10_IMGPII45 & SQS & 3260,0 & IMGPII45 & Pop-in Monitor Type II-45 \\
\hline$T 10$ & XTD10 & & & SQS & 3265,6 & IMGPIII & OPTION: Pop-in Monitor Type III \\
\hline$T 10$ & XTD10 & IMGES.3319.T10.SQS & SQS_XTD10_IMGES & SQS & 3319,3 & IMGES & Exit Slit Imager \\
\hline T10 & XTD10 & IMGPI.3334.T10.SQS & SQS XTD10_IMGPI & SQS & 3334,3 & IMGPI & Pop-in Monitor Type I \\
\hline T10 & XTD10 & IMGES.3319.T10.SCS & SCS_XTD10_IMGES & SCS & 3319,3 & IMGES & Exit Slit Imager \\
\hline T10 & XTD10 & IMGPI.3334.T10.SCS & SCS XTD10_IMGPI & SCS & 3334,3 & IMGPI & Pop-in Monitor Type I \\
\hline$T 10$ & XTD10 & & & SQS2 & 3319,3 & IMGES & Exit Slit Imager \\
\hline T10 & XTD10 & IMGPI.3334.T10.SQS2 & SQS2_XTD10_IMGPI & SQS2 & 3334,3 & IMGPI & Pop-in Monitor Type I \\
\hline \multicolumn{8}{|c|}{ Experimental hall } \\
\hline & XHEXP1 & XGM.3348.T10.SCS & SCS_BLU XGM & SCS & 3348,0 & $\mathrm{XGM}$ & X-ray Gas Monitor \\
\hline & XHEXP1 & TPLM.3348.T10.SQS & SQS_DIAG3_TPLM & SQS & & TPLM & TPLM prototype \\
\hline & XHEXP1 & PAM.3348.T10.SCS & SPB_EHC_PAM & SPB/SFX & & PAM & PAM prototype \\
\hline & distributed & & & & & & Gas supply system \\
\hline
\end{tabular}

Figure 2

Photon diagnostics assemblies throughout the facility. The longitudinal positions are given in linear accelerator (LA) coordinates. Undulator and sourcepoint positions are given for reference. 
temporal diagnostics (Liu, 2015; Liu et al., 2017) employed to monitor the X-ray pulse arrival time, pulse duration and, ideally, the full temporal shape as shown in the literature (Maltezopoulos et al., 2008; Grguras et al., 2012; Harmand et al., 2013; Hartmann et al., 2014). Wavefront measurements and coherence monitors are also located in the scientific instruments. These devices are described elsewhere.

At the time of this writing in fall 2018, the photon diagnostics systems in all tunnels have been installed, technically commissioned, initially commissioned with beam and used in standard operation. As the entire machine gradually expands its operational capabilities towards the full design parameters, our diagnostics will be further commissioned in parallel at each new machine capability step:

(i) Increases in the number of pulses per train: tested with up to 500 pulses per train in SASE1 and 300 pulses per train in SASE3.

(ii) Changes of repetition rates within the pulse trains: 4.5 MHz, 1.1 MHz and $550 \mathrm{kHz}$. tion.

(iii) Special bunch patterns beyond periodic pulse repeti-

(iv) Increase of photon energy: in SASE1, explored range 7-14 keV; in SASE3, explored 700-1200 eV.

\section{Online photon diagnostics}

These systems can be separated into gas-based systems, with only minimal interference with the X-ray beam, and systems using very thin solid films or crystals, thereby absorbing a minor fraction of the FEL pulse. The latter method is applicable only for hard X-ray radiation, otherwise the X-ray absorption is prohibitively high.

In the gas-based diagnostic systems, photoionization of rare gases $(\mathrm{Xe}, \mathrm{Kr}, \mathrm{Ne}$ ) or nitrogen is utilized. Therefore, these devices are indestructible and highly transparent (Tiedtke et al., 2008; Sorokin et al., 2011). These non-invasive diagnostic methods are best suited for high peak energies and high average flux since there is no damage or heating due to the absorbed X-ray pulse energy and no influence on the wavefront or coherence of the transmitted pulses. At the European XFEL, these systems are employed in the beam transport to measure pulse energy, beam position, spectrum and polarization of the X-ray pulses. Gas monitors can operate continuously up to very high pulse repetition rates, limited only by the flight time of ions and electrons used for the measurement. They also work for hard X-rays if a sufficient sensitivity is provided to compensate for the reduced cross-sections. As of today, no reports have been made about distortion of coherence and wavefront properties due to gas monitors. However, for the highest repetition rates, number of pulses per train and elevated gas pressures, depletion of the gas column could occur (Feng \& Raubenheimer, 2017).

Online solid-state systems employ either thin foils to scatter a fraction of the X-ray beam, using the detection of this scattered fraction to measure the pulse energy and, in a special configuration, beam position (Tono et al., 2011), or thin curved crystals to disperse the incident spectrum on a position- sensitive detector (Zhu et al., 2012). In both cases, only a small fraction of the X-ray beam is absorbed or scattered; however, these systems face limitations at very high pulse energies and a large number of pulses in a pulse train. In particular, limited heat transport is a concern at high repetition rates.

\subsection{X-ray gas monitors}

The X-ray gas monitors (XGMs) are online pulse energy (photon flux) and beam position monitors that resolve individual photon pulses at $\mathrm{MHz}$ rates (temporal resolution of detection better than $100 \mathrm{~ns}$; Tiedtke et al., 2008). Due to a gain of up to $10^{6}$, individual $\mathrm{X}$-ray pulses with femtosecond durations containing from $10^{7}$ up to $10^{15}$ photons can be measured with better than $10 \%$ absolute accuracy and with $\leq 1 \%$ relative (pulse-to-pulse) accuracy for pulses with more than $10^{10}$ photons. Beam position is monitored in both transverse directions with accuracy on the order of $\pm 10 \mu \mathrm{m}$ within a range of $\pm 1 \mathrm{~mm}$. There is an XGM in the direct beam of each undulator, monitoring the source properties upstream of the double horizontal offset mirror systems. Three more XGMs are placed upstream of the scientific instruments SPB/SFX, Spectroscopy and Coherent Scattering (SCS), and High Energy Density (HED), i.e. one per undulator branch, to monitor the pulse properties actually delivered to the experiments after passing several X-ray optics elements in the tunnels.

The design of these monitors is a joint development of the Deutsches Elektronen-Synchrotron (DESY) and European XFEL, based on gas monitor detectors previously developed and operating at the Free-electron LASer in Hamburg (FLASH) user facility. The main special requirement at the European XFEL was the significantly higher photon energy up to $24 \mathrm{keV}$ in the fundamental. This is accommodated by a new detector: Huge Aperture open Multiplier (HAMP), which was also developed and constructed in-house at DESY by the FLASH team. Therefore, each XGM consists of four vacuum chambers on a common girder, where two of them contain the XGMDs and two of them the new HAMPs. The XGMDs measure the single-shot-resolved photoelectron signal and averaged absolute calibrated photo-ion signal. The HAMPs measure the single-shot-resolved photo-ion signal, albeit at higher sensitivity for harder X-rays.

Each chamber contains a pair of diagonally split 'backgammon' electrodes to measure the X-ray beam position in one transverse direction. There are two chambers of each version rotated by $90^{\circ}$ to each other to enable beam position measurements in both transverse directions. The variety of signals offered by one such XGM provides great flexibility to cover the extensive wavelength range from a few $100 \mathrm{eV}$ to beyond $20 \mathrm{keV}$. Typically, we operate the hard X-ray units with xenon at partial pressures of $10^{-6}$ mbar to $10^{-4} \mathrm{mbar}$, and the soft X-ray units with krypton or neon at similar pressures depending on the achieved maximum pulse intensities and the number of pulses per train. In all tunnels, four gases (purity 5.0) are available through a distributed gas supply system. It connects gas bottles in cabinets outside the tunnels via $4 \mathrm{~km}$ of 
$6 \mathrm{~mm}$-diameter stainless steel tubes with the gas distribution panels at each online diagnostics gas consumer station. For purity reasons, each gas has its own line and is never exchanged. Currently, we provide xenon, krypton, neon and nitrogen. Changing the gas type is mandatory when working at wavelengths close to an absorption edge. Due to the small gas flux and the intrinsic chemical passiveness of these gases, there is no need for recovery systems; however, the pressures in all lines and bottles are remotely and continuously monitored. Having the bottles outside the access restricted radiation safety tunnel areas enables bottle exchange during full beam operation and removes safety hazards from the underground tunnels.

Since the XGMs are crucial for machine operation and regularly used for SASE tuning by the accelerator operators, their data acquisition software was implemented in the machine control system DOOCS. The local processing unit in the tunnel calculates per-pulse properties from the raw ADC signal and then provides this pulse-resolved XGM data to the network in one collective per-train dataset at $10 \mathrm{~Hz}$. The XGM vacuum control was implemented in the photon system controls (Beckhoff PLC and Karabo high-level controls; Hauf et al., 2019). More recently, the XGM data were also made available in Karabo via so-called 'bridge software devices'. Therefore, the scientific instruments can record data from their area detectors directly together with pulse-resolved XGM data.

Each XGMD provides integrational signals: the photocurrent on bare electrodes measured by picoammeters (Keithley 6514E) with a time constant of $\sim 30$ s. This signal was absolutely calibrated with synchrotron radiation for each XGM unit at Helmholtz-Zentrum Berlin (HZB; MLT-beamline of PTB). Additionally, there are the pulse-resolved signals: each XGMD chamber provides a fast photo-electron signal collected at bare detection plates, and one of the two XGMD chambers provides additionally a photo-ion signal detected by a small aperture commercial multiplier (ETP).

The pulse-resolved signals are processed by fast DAQ boards (Teledyne Signal Processing Devices ADQ412, 3G MTCA, $2 \mathrm{GHz}$ bandwidth) and cross-calibrated to the integrational signals from the Keithleys. For this purpose, a signal with a comparable time response is continuously calculated by taking a rolling average of the pulse-resolved data.

During SASE tuning, the machine operators use the raw signal of a HAMP detector, which is sensitive to even the lowintensity levels of spontaneous radiation from a few undulator segments. A typical signal trace is shown in Fig. 3.

To a large extent, the required adaptation of operational parameters and signal processing are performed automatically so that $24 / 7$ operation is possible. Four XGMs are already continuously operating, and two more are under commissioning. So far, the XGMs were used in FEL operation with up to 500 pulses per train at $4.5 \mathrm{MHz}$, with single-pulse intensities up to $2 \mathrm{~mJ}$ at $9-14 \mathrm{keV}$ and $4 \mathrm{~mJ}$ at $700-900 \mathrm{eV}$. Fig. 4 shows a typical XGM dataset during long bunch-train delivery with 300 pulses per train at $1.1 \mathrm{MHz}$ repetition rate $(700 \mathrm{eV})$. The intensity variation averaged over the last 500 trains is

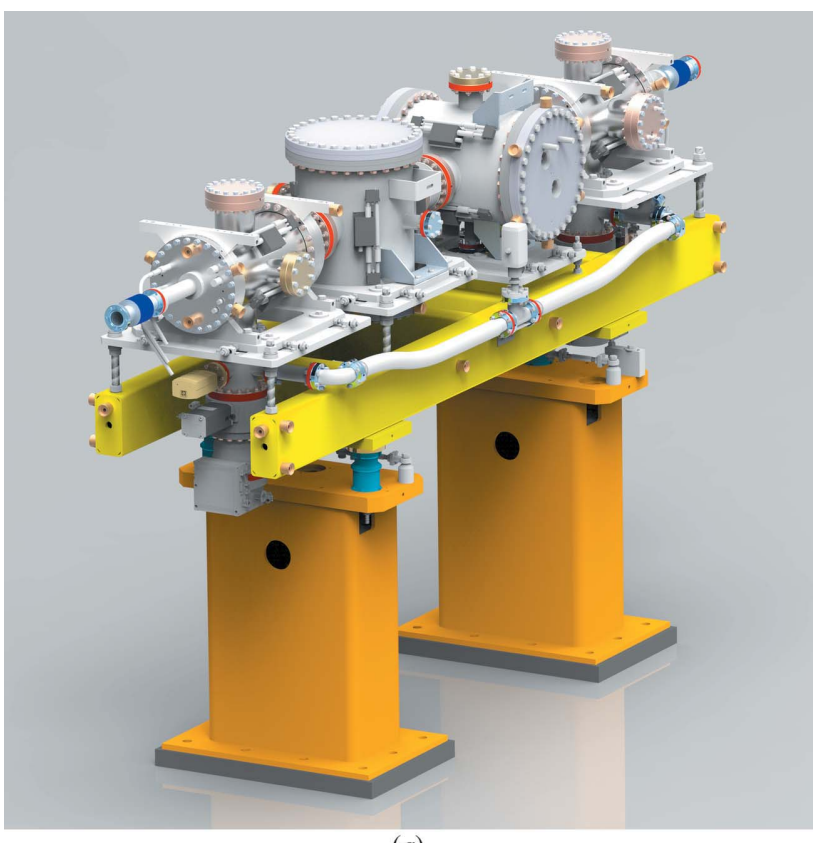

(a)

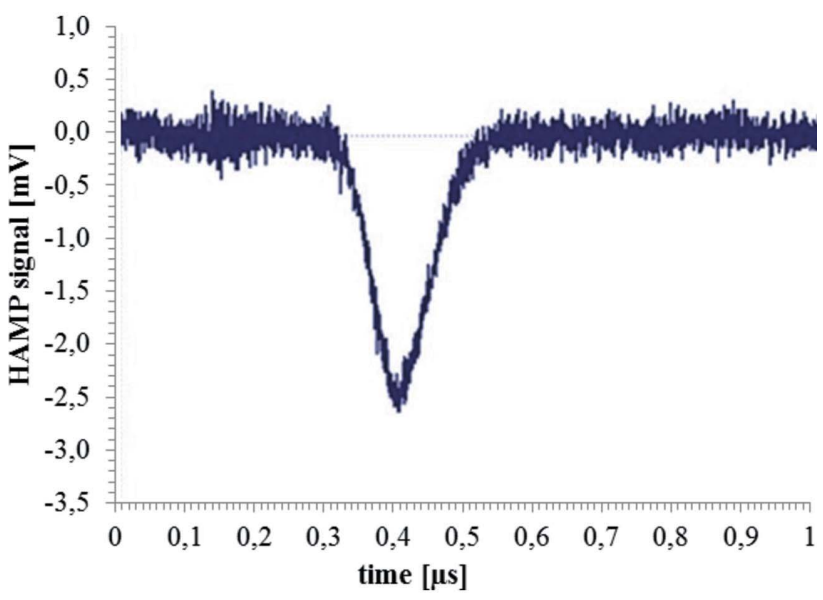

(b)

Figure 3

(a) CAD representation of the XGM. Here the unit in SASE1/XTD2 is shown. Infrastructure such as cables, local electronics, gas supply lines, air pressure lines and pre-vacuum equipment is not shown for clarity. (b) Typical raw signal trace of the pulse-resolved HAMP detector.

continuously displayed and ranges between $5 \%$ and $10 \%$ when the machine is well tuned.

The average beam position is monitored online and will be used to steer the mirrors in a feedback loop for beam position stabilization at the scientific endstations.

\subsection{High-resolution hard X-ray single-pulse diagnostic spectrometer}

The high-resolution hard X-ray single-pulse diagnostic spectrometer (HIREX) is an online device based on a transmission diamond diffraction grating to split off a small fraction $(0.1 \%)$ of the photon beam, a bent crystal as a dispersive element and a $\mathrm{MHz}$ repetition rate 1D strip detector. Before implementation at the European XFEL, we verified the 


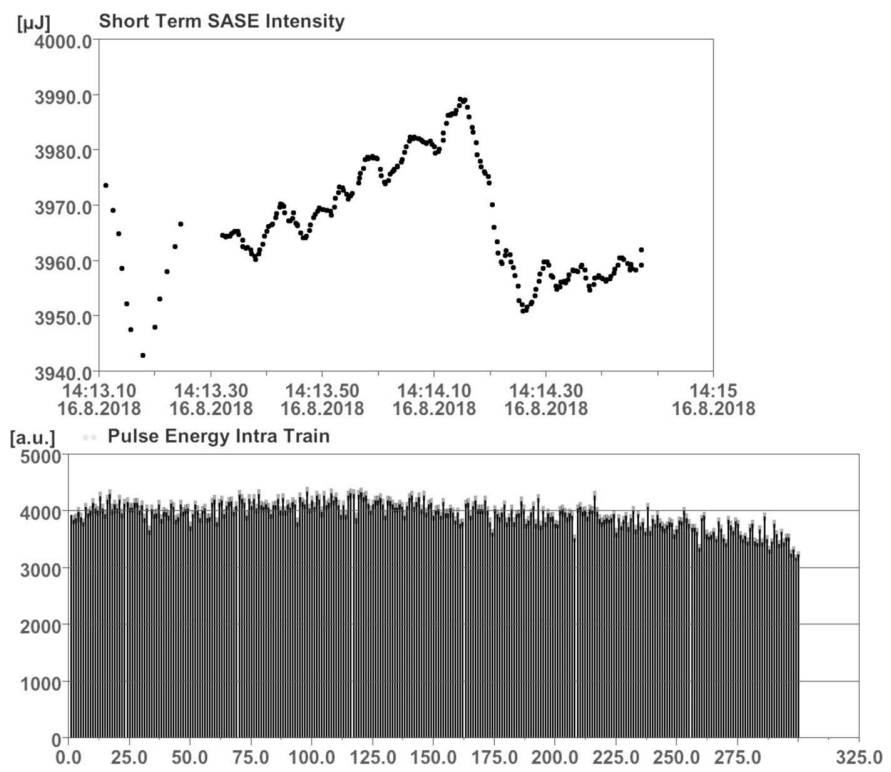

Figure 4

XGM measurements during a material test with high pulse intensity ( $\sim 4 \mathrm{~mJ}$ per pulse) and long pulse trains (300 pulses per train): average per pulse intensity based on the slow absolute calibrated ion signal (top graph); intra-train pulse-resolved intensity based on the fast ADC signal (bottom graph).

working principle of this spectrometer setup during user beam time (Makita et al., 2015). The HIREX spectrometer is installed in the XTD9 tunnel of the SASE1 beamline.

Fig. 5 shows a CAD drawing of the two main vacuum chambers: the grating and crystal units. Two gratings with pitches of $150 \mathrm{~nm}$ and $200 \mathrm{~nm}$ were fabricated by the Nanolab at the Paul Scherrer Institute (PSI), Switzerland (Makita et al., 2017). These gratings are installed in the grating chamber on a linear manipulator. The 0th-order beam is delivered to the scientific endstation and the 1st-order beam is used for spec- tral measurement. Three silicon crystals $(10 \mu \mathrm{m}$ thickness $)$ with fixed bending radii of $50 \mathrm{~mm}, 75 \mathrm{~mm}$ and $100 \mathrm{~mm}$, and with orientations 110 and 111 , are installed in the crystal chamber. The crystals are mounted on a rotation stage that allows the Bragg condition to be achieved. The distance between gratings and crystals is $10 \mathrm{~m}$ in order to obtain sufficient separation between the 0th- and 1st-order diffracted beams. While the beam transmission depends on the photon energy, typically a $95 \%$ transmission is achieved and the remaining $5 \%$ of the pulse energy is spread over all diffraction orders. The vertical orientation of the grating lines leads to diffraction into the horizontal plane. The 1st-order diffracted beam is sent from the grating towards one of the bent crystals for energy dispersion into the vertical plane under the Bragg condition (Zhu et al., 2012). Two detectors are available for data acquisition: an optical sCMOS camera (Photonic Science) for full transverse 2D imaging at low repetition rate, and a modified Gotthard-II 1D strip detector (PSI) for fast data acquisition at $4.5 \mathrm{MHz}$.

Fig. 6 presents a single-shot spectrum from the Si 110 crystal with a bending radius of $100 \mathrm{~mm}$ in the 220 reflection. Data were collected in single-pulse mode with the Si crystal placed into the 0th-order beam at a photon energy of $9.3 \mathrm{keV}$. The machine was set to $14 \mathrm{GeV}$ electron energy and a charge of $0.25 \mathrm{nC}$. Fig. $6(a)$ shows the single-shot spectrum at a pulse intensity of $1 \mathrm{~mJ}$ with the energy dispersion in the vertical direction and the beam intensity profile in the horizontal direction. Fig. 6(b) shows the spectrum derived from the camera image as a line profile along the energy dispersive direction. The data were acquired with a Photonic Science camera.

\section{Invasive photon diagnostics systems}

The invasive diagnostics are either used for initial commissioning with spontaneous radiation (synchrotron radiation)

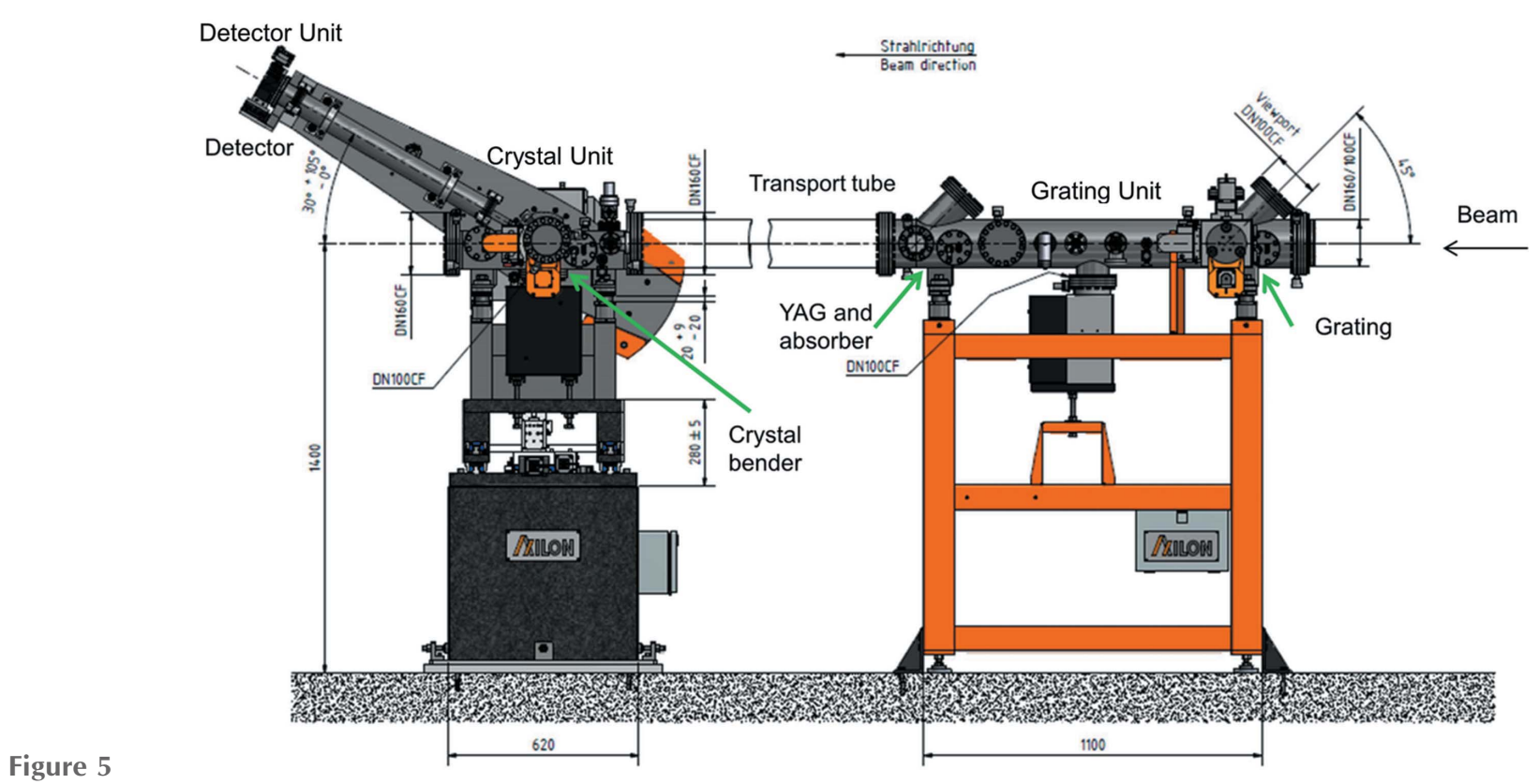

CAD drawing of the HIREX spectrometer installed in the SASE1 beamline in tunnel XTD9. 


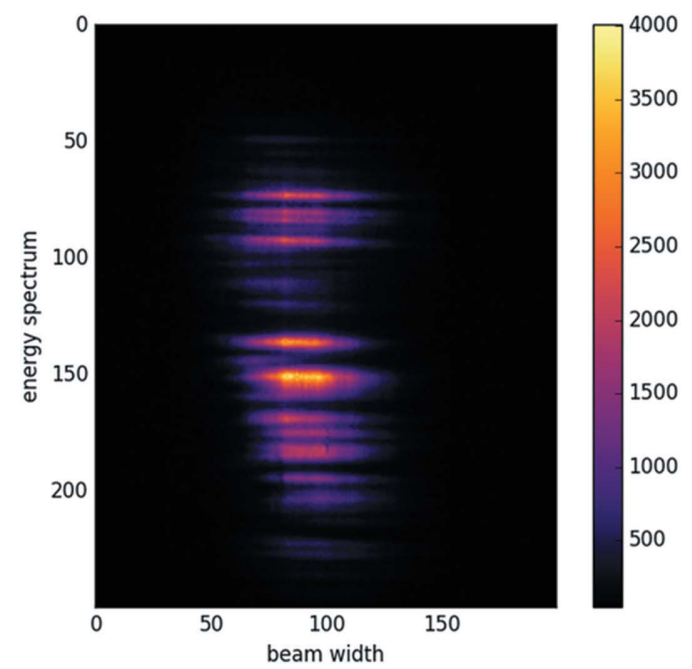

(a)

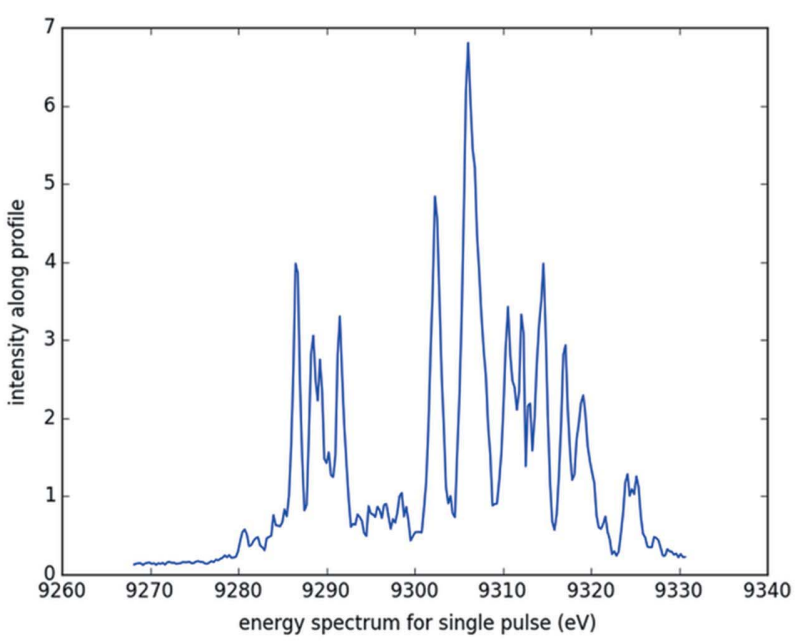

(b)

Figure 6

Single-shot spectra were acquired with the Si 110 crystal in 220 reflection with a fixed bending radius of $100 \mathrm{~mm}$ at $9.3 \mathrm{keV}$ (fundamental) and a Photonic Science camera was used to collect spectra, with an electron beam energy of $14 \mathrm{GeV}$, in single-pulse mode. (a) Camera image (the axis values are number of pixels). The $y$ direction contains the spectral information. (b) Line profile along the energy dispersion direction of the camera image.

for FEL commissioning, or for setup purposes prior to and during measurements.

\subsection{MCP-based detector}

When all undulator segments are inserted to establish the SASE conditions for lasing, this detector (Syresin et al., 2011, 2014; Syresin, 2012) measures intensities from the initial signs of lasing up to saturation. Two horizontal manipulators insert either $15 \mathrm{~mm}$-diameter MCP discs for integral intensity monitoring with $1 \%$ relative accuracy over a large pulse energy range $(1 \mathrm{~nJ}$ to $10 \mathrm{~mJ})$ and a photodiode (Hamamatsu, $10 \mathrm{~mm} \times 10 \mathrm{~mm}, 300 \mu \mathrm{m}$-thick), or a larger MCP-intensified phosphor screen (BOS-MCP, Beam Imaging Systems) providing an intensified beam image with $30 \mu \mathrm{m}$ resolution via an optical camera setup. The integrated MCPs are a tool for the operators during the SASE tuning process in any of the three SASE beamlines. The MCPs in SASE3 provide fully parasitic pulse-resolved intensity monitoring: when positioned close but outside of the direct FEL beam, these MCPs can owing to their high sensitivity - detect an FEL-correlated signal due to scattered light from the offset mirrors, despite the strong synchrotron radiation background. Additionally, the MCP detector in SASE3 is crucial for studies on gas attenuator efficiency depletion because it is the only pulseresolved intensity monitor for high repetition rates in the tunnel downstream of the gas attenuator.

\subsection{Undulator commissioning spectrometer}

The undulator $K$ parameter can be determined with photon beam based methods (Welch et al., 2009; Tanaka et al., 2012) by analysing the spontaneous radiation from a single or a few undulator segments with a suitable monochromator and detector. This analysis is an independent and in situ verification of the magnetic measurements performed in the undulator laboratory and is necessary in order to set all undulator segments to $\Delta K / K<10^{-4}$ and to adjust the phase shifters between them. Our implementation of this technique (Freund, 2011; Ozkan et al., 2012) involves a monochromator ('Kmono'), which contains two monolithic Si channel-cut crystals that can be used in two- or four-bounce geometry. The Bragg angle range from $7^{\circ}$ to $55^{\circ}$ covers a photon energy range of 2.5-16 keV with Si (111) and 7.5-48 keV with Si (333). The energy resolution $\Delta E / E$ is $2 \times 10^{-4}$ for $\mathrm{Si}(111)$ and $10^{-5}$ for Si (333). The crystals can be retracted from the beam in the horizontal direction. The diffracted X-rays are detected by a photodiode $(10 \mathrm{~mm} \times 10 \mathrm{~mm}$ active area) or a highly sensitive imager (see the synchrotron radiation imager in Section 3.3). Upstream of the K-mono, there is a filter chamber that contains foils of $\mathrm{Mo}, \mathrm{Cu}, \mathrm{Ni}$ and $\mathrm{Al}$ with $30 \mathrm{~mm}$ diameters and varying thicknesses for the purpose of spectral calibration by scanning across the filter $K$-edges and blocking optical light (3 $\mu \mathrm{m} \mathrm{Al})$. The overall setup is shown in Fig. 7 .

The K-mono, in combination with the synchrotron radiation imager, gives the machine operators an intuitive way of measuring in real time the angular pointing and $K$-parameter of single undulator segments. This information can be used for orbit corrections, which has increased the contribution to lasing of the last five SASE1 undulator segments.

A more fundamental K-mono measurement is the determination of the absolute $K$ value of an individual segment. This determination is realized by recording a spectral scan with the photodiode, either by rotating the K-mono crystal or by changing the undulator gap. The minimum of the derivative of this signal reveals the steepest slope which is the highenergy edge of the spontaneous radiation. Together with the measured electron beam energy this determines the $K$ value (Freund et al., 2018). An example of this measurement mode is shown in Fig. 7(b) where segment \#36 of SASE1 was studied. Precise energy calibration is possible owing to 'glitches' in the scan, which can be identified as higher Bragg reflections with known energy. Fig. 8 shows the interesting spatial radiation 


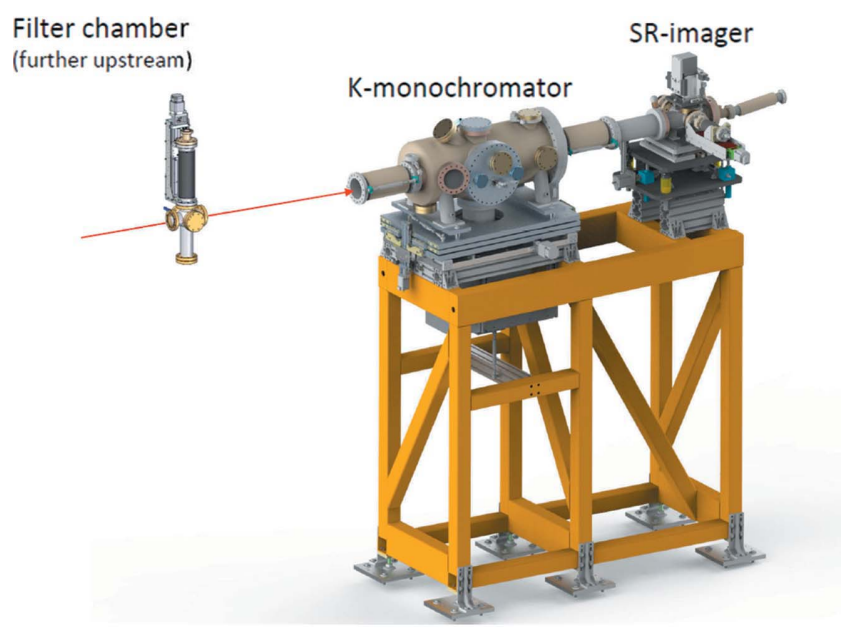

(a)

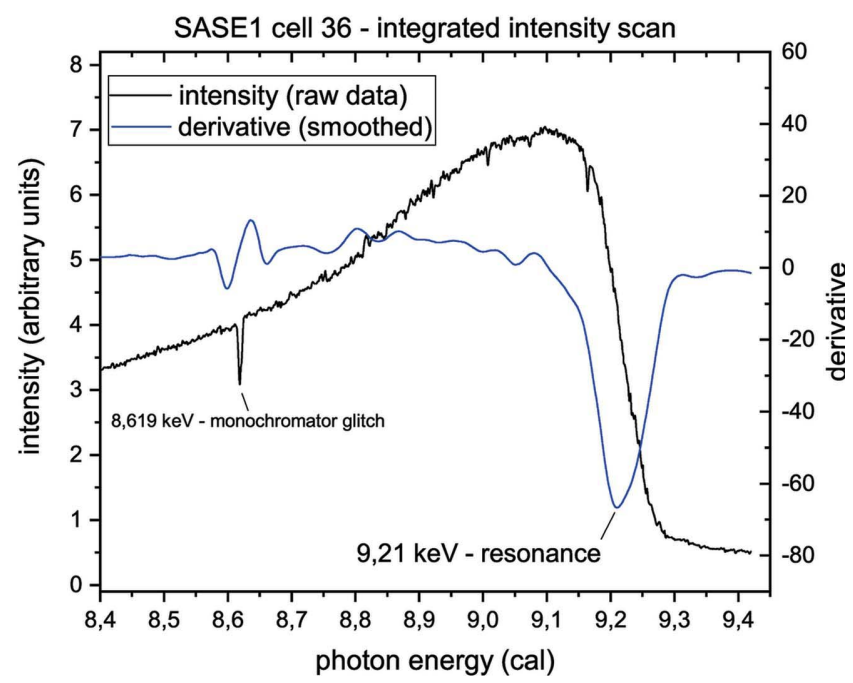

(b)

Figure 7

(a) Schematic view of the K-monochromator system. (b) Spectral scan recorded by a photodiode using segment \#36 of SASE1.

distribution of high harmonics (here the 12th and 17th harmonic of a soft X-ray fundamental at $900 \mathrm{eV}$ ) observed with the K-mono system. The dark stripe in the lower part of the image is caused by the decreasing acceptance aperture of the analyser channel-cut crystal at the shallow Bragg angles required for higher harmonics.

In addition to the measurements with synchrotron radiation, studies with FEL radiation were also performed in the hard X-ray beamlines (not possible at SASE3) by scanning the monochromator energy. In this way, the average spectral bandwidth and the central photon energy of the FEL can be determined.

\subsection{Imagers}

There are 25 imaging units distributed over the photon tunnels that serve different purposes and therefore have different resolutions, fields of view (FOV) and geometries (Ozkan, 2012; Ozkan et al., 2012; Koch et al., 2015). All imagers contain one or more scintillators, mostly YAG:Ce,

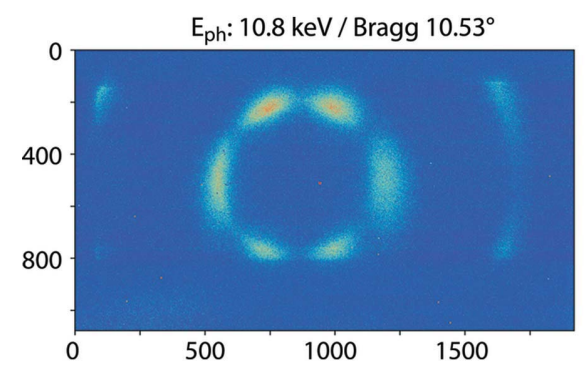

(a)

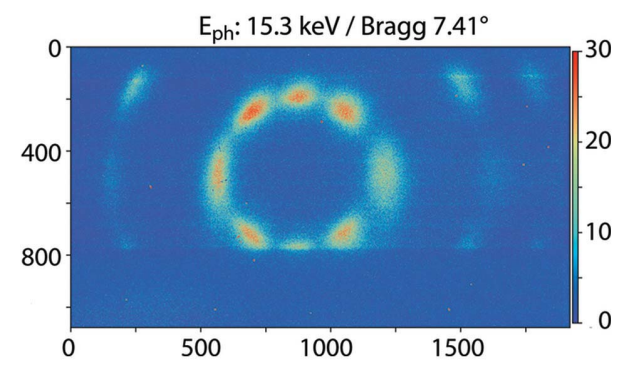

Figure 8

(b)

Images of $(a)$ the 12th-harmonic and $(b)$ the 17th-harmonic spontaneous radiation from the most downstream undulator segment in SASE3 at $900 \mathrm{eV}$ fundamental. The imager is $189 \mathrm{~m}$ from this segment. The axis values correspond to the number of pixels with $14 \mu \mathrm{m}$ pixel size.

sometimes additionally polycrystalline diamond, and all but one type has stationary optics with sCMOS GigE cameras and fixed-focus lenses (see, for example, Fig 9). There are the following imaging stations:

(i) Transmissive imagers (one per FEL) are closest to the source and have the thinnest scintillators to allow transmition of the beam for recording another image of the same photon pulse at a downstream imager. With this method, beam pointing and beam offset data can be obtained simultaneously.

(ii) The synchrotron radiation imagers (one per FEL) are optimized for highest photon sensitivity to detect spontaneous radiation from single undulator segments when applied toge-

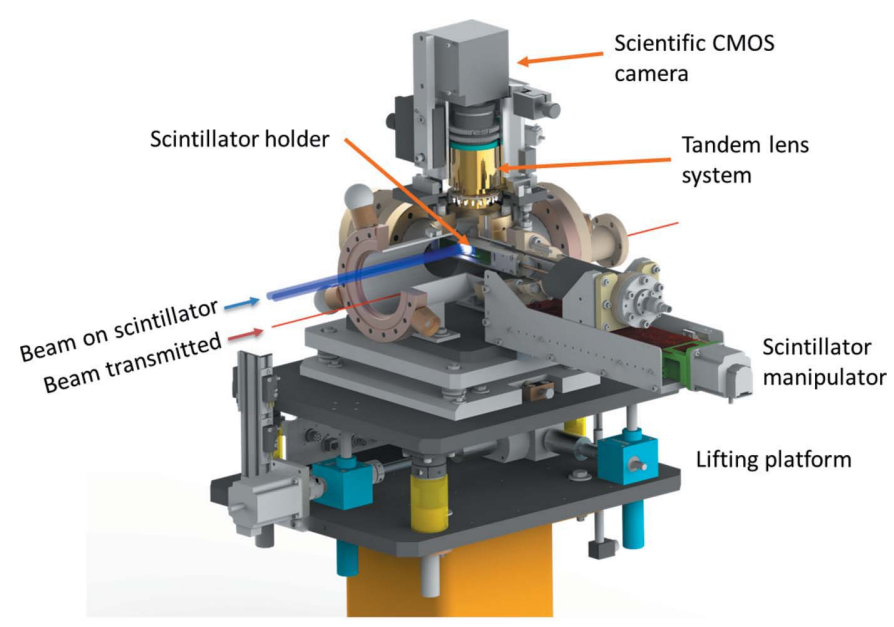

Figure 9

CAD model of the SR imager

Sketch of a synchrotron radiation imager with a cut-view into its vacuum chamber 


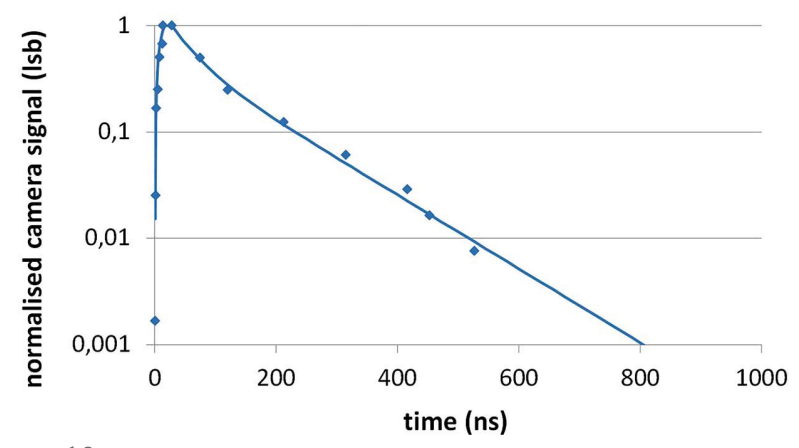

Figure 10

Time response of the gated exit slit imager (YAG:Ce screen), measured data points and fitted curve, one bunch per train, $900 \mathrm{eV}, 100 \mu \mathrm{J}$. MCP gate opening time is $19 \mathrm{~ns}$.

ther with the K-mono system in undulator commissioning and photon beam based alignment. The synchrotron radiation imagers have an optical resolution of $25 \mu \mathrm{m}$ full width at halfmaximum (FWHM) and a FOV of $26.6 \mathrm{~mm} \times 15 \mathrm{~mm}$ using YAG:Ce and ceramic $\mathrm{Gd}_{2} \mathrm{O}_{2} \mathrm{~S}: \operatorname{Pr}$ (GOS) scintillators. The noise equivalent signal with the GOS scintillator is 2 photons of $12 \mathrm{keV}$. Fig. 8 shows example datasets acquired with this imager.

(iii) The FEL imagers (one per FEL) are optimized for detailed and precise spatial characterization of the FEL beam to quantify the transverse intensity profile with beam position, size and shape. Their optical resolution is $15 \mu \mathrm{m}$ (FWHM) and the FOV is $16 \mathrm{~mm} \times 22 \mathrm{~mm}$. These imagers have redundancy scintillators of several different materials.

(iv) Pop-in monitors (15 in total) are the basic imagers for beam finding and alignment. These monitors are placed downstream of major optical elements such as mirrors and monochromators. Their horizontal FOV is large enough to cover the variable beam offset without movements of the scintillator or optics. Various geometries are employed. Most devices put the scintillator at $45^{\circ}$ to the X-ray FEL beam, but some have the scintillator at normal incidence with an additional optical mirror. Optical resolutions range from $38 \mu \mathrm{m}$ to $120 \mu \mathrm{m}(\mathrm{FWHM})$ and FOVs range from $22.7 \mathrm{~mm}(\mathrm{~h}) \times 40 \mathrm{~mm}$ (v) up to $189 \mathrm{~mm}(\mathrm{~h}) \times 22 \mathrm{~mm}(\mathrm{v})$, also with a wider view in the vertical direction $93 \mathrm{~mm}(\mathrm{~h}) \times 118 \mathrm{~mm}(\mathrm{v})$.

(v) Exit slit imagers are installed on the two exit slits of the SASE3 monochromator for beam alignment but, more importantly, to deliver single-pulse soft X-ray spectra with a resolution $\Delta E / E \geq 10^{-5}$. The imager provides single-pulse gating per bunch train by using an MCP in the optical system. The limiting component for the time response is a YAG:Ce screen. The response curve is shown in Fig. 10. A crosstalk of $10 \%$ is expected for successive pulses at a $4.5 \mathrm{MHz}$ repetition rate. This result is relevant for the design of future imager upgrades in all beamlines.

\section{Summary and future developments}

The baseline photon diagnostic devices of the European XFEL were installed, commissioned and have started operation. They were used to prepare, support and detect first lasing, to commission the beam transport and optics, and to deliver beam to the scientific instruments. This was first achieved for the SASE1 undulator in the hard X-ray domain and then for undulators SASE2 and SASE3. More details about diagnostics for the SASE2 and SASE3 beamlines will be presented in further publications. Diagnostics of the photon beam continues in this commissioning phase with remarkable qualitative progress each time the electron beam machine achieves new capabilities towards the design parameters, such as increasing the number of bunches per bunch-train, increasing the photon energy and providing special ondemand bunch patterns. New capabilities of the installed diagnostics were activated based on the actual machine operation status and data acquisition system availability. The online gas-based intensity monitor operates continuously $24 / 7$ and is almost entirely automated. It is also used to characterize individual pulses, so far with up to 500 pulses per train.

In future work, existing types of instrumentation, such as the soft X-ray photoelectron spectrometer (PES), will be modified for and applied to new photon energy ranges. More imagers can be equipped with single-pulse gating as pioneered at the exit slit imager or with high-speed cameras for successive image recording at $4.5 \mathrm{MHz}$. New types of instrumentation for temporal diagnostics, such as photon arrival monitors (PAMs) and THz-based pulse duration monitors (TPLMs), and diamond detectors (Roth et al., 2018) as well as wavefront sensors are under construction and will be installed in the scientific instruments.

\section{Acknowledgements}

All contributors to European XFEL photon diagnostics, both internal and external, are acknowledged for their outstanding contributions. The group of Kai Tiedtke at FLASH/DESY, Hamburg, Germany, developed and constructed the XGMs. The team of Evgeny Syresin at JINR, Dubna, Russia, provided the MCP-based detectors. The commissioning of the photon system is a collaborative effort of several European XFEL groups. It is performed by the Photon Diagnostics Team together with the Photon Commissioning Team, the latter being organized by Harald Sinn. The X-Ray Photon Diagnostics group worked closely with the other European XFEL scientific support groups: X-Ray Optics (XRO) led by Harald Sinn, Vacuum (VAC) led by Martin Dommach, Advanced Electronics (AE) led by Patrick Gessler, Information Technology and Data Management (ITDM) led by Krzystof Wrona, and Control and Analysis Software (CAS) led by Sandor Brockhauser. From DESY, the Survey and Alignment team led by Johannes Prenting, the EMV team led by Herbert Kapitza, the transport and infrastructure groups MEA and MKK, and the DOOCS specialists at MCS gave valuable support. We would like to thank all these groups for their continuous efforts. Author Contributions: JG conceived the article; WF, AK, NK, JiL, JoL and TM contributed to this paper; all authors were involved in the installation, commissioning or operation of the described diagnostics devices. The authors declare no conflict of interest. 


\section{References}

Altarelli, M., Brinkmann, R., Chergui, M., Decking, W., Dobson, B., Düsterer, S., Grübel, G., Graeff, W., Graafsma, H., Hajdu, J., Marangos, Jonathan., Pflüger, J., Redlin, H., Riley, D., Robinson, I., Rossbach, J., Schwarz, A., Tiedtke, K., Tschentscher, T., Vartaniants, I., Wabnitz, H., Weise, H., Wichmann, R., Witte, K., Wolf, A., Wulff, M. \&Yurkov M. (2006). The European X-ray Free-Electron Laser Technical Design Report. DESY Report 2006-097. DESY, Hamburg, Germany.

Feng, Y. \& Raubenheimer, T. O. (2017). Proc. SPIE, 10237, 1023714.

Freund, W. (2011). European XFEL Technical Note. XFEL.EU TN-2014-001-01. European XFEL Facility, Hamburg, Germany.

Freund, W., Grünert, J., Liu, J., Karabekyan, S. \& Koch, A. (2018). Proceedings of the 13th International Conference on Synchrotron Radiation Instrumentation (SRI2018), 11-15 June 2018, Taipei, Taiwan. AIP Publishing. In the press.

Galler, A., Gawelda, W., Biednov, M., Bomer, C., Britz, A., Brockhauser, S., Choi, T-K., Diez, M., Frankenberger, P., French, M., Görries, D., Hart, M., Hauf, S., Khakhulin, D., Knoll, M., Korsch, T., Kubicek, K., Kuster, M., Lang, P., Lima, F., A., Otte, F., Schulz, S., Zaldena, P. \& Bresslera, C. (2019). J. Synchrotron Rad. 26, 1432-1447.

Grguras, I., Maier, A. R., Behrens, C., Mazza, T., Kelly, T. J., Radcliffe, P., Düsterer, S., Kazansky, A. K., Kabachnik, N. M., Tschentscher, Th., Costello, J. T., Meyer, M., Hoffmann, M. C., Schlarb, H. \& Cavalieri, A. L. (2012). Nat. Photon. 6, 852-857.

Grünert, J., Koch, A., Kujala, N., Freund, W., Planas, M., Dietrich, F., Buck, J., Liu, J., Sinn, H., Dommach, M. \& Molodtsov, S. (2015). Proceedings of the 37th International Free Electron Laser Conference (FEL 2015), 23-28 August 2015, Daejeon, South Korea. WED03.

Grünert, J. (2012). European XFEL Technical Report, XFEL.EU TR-2012-003. European XFEL Facility, Hamburg, Germany.

Grünert, J., Buck, J., Freund, W., Ozkan, C. \& Molodtsov, S. (2013). J. Phys. Conf. Ser. 425, 072004.

Grünert, J., Buck, J., Ozkan, C., Freund, W. \& Molodtsov, S. (2012). Proc. SPIE, 8504, 8504OR.

Grünert, J., Carbonell, M. P., Dietrich, F., Freund, W., Koch, A., Kujala, N., Laksman, J., Liu, J. \& Maltezopoulos, T. (2018). AIP Conf. Proc. 2054, 030014.

Harmand, M., Coffee, R., Bionta, M. R., Chollet, M., French, D., Zhu, D., Fritz, D. M., Lemke, H. T., Medvedev, N., Ziaja, B., Toleikis, S. \& Cammarata, M. (2013). Nat. Photon. 7, 215-218.

Hartmann, N., Helml, W., Galler, A., Bionta, M. R., Grünert, J., Molodtsov, S. L., Ferguson, K. R., Schorb, S., Swiggers, M. L., Carron, S., Bostedt, C. Castagna, J.-C., Bozek, J., Glownia, J. M., Kane, D. J., Fry, A. R., White, W. E., Hauri, C. P., Feurer, T. \& Coffee, R. N. (2014). Nat. Photon. 8, 706-709.

Hauf, S., Heisen, B., Aplin, S., Beg, M., Bergemann, M., Bondar, V., Boukhelef, D., Danilevsky, C., Ehsan, W., Essenov, S., Fabbri, R., Flucke, G., Marsa, D. F., Goeries, D., Giovanetti, G., Hickin, D., Jarosiewicz, T., Kamil, E., Khakhulin, D., Klimovskaia, A., Kluyver, T., Kririenko, Y., Kuhn, M., Maia, L., Mamchyk, D., Mariani, V., Mekinda, L., Michelat, T., Muennich, A., Padee, A., Parenti, A., Santos, H., Silenzi, A., Teichmann, M., Weger, K., Wiggins, J., Wrona, K., Xu, C., Youngman, C., Zhu, J., Fangohr H. and Brockhauser, S. (2018). J. Synchrotron Rad. 26, 1448-1461.

Koch, A., Freund, W., Gruenert, J., Planas, M., Roth, T., Samoylova, L. \& Lyamayev V. (2015). Proc. SPIE, 9512, 95121R1.

Liu, J. (2015). European XFEL Technical Note, XFEL.EU TN-2015002-01. European XFEL Facility, Hamburg, Germany.

Liu, J., Dietrich, F. \& Grünert, J. (2017). European XFEL Technical Report, XFEL.EU TR-2017-002. European XFEL Facility, Hamburg, Germany.
Makita, M., Karvinen, P., Guzenko, V. A., Kujala, N., Vagovic, P. \& David, C. (2017). Microelectron. Eng. 176, 75-78.

Makita, M., Karvinen, P., Zhu, D., Juranic, P., Grünert, J., Cartier, S., Jungmann-Smitz, J. H., Lemke, H. T., Mozzanica, A., Nelson, S., Patthey, L., Sikorski, M., Song, S., Feng, Y. \& David, C. (2015). Optica 2, 10.

Maltezopoulos, Th., Cunovic, S., Wieland, M., Beye, M., Azima, A., Redlin, H., Krikunova, M., Kalms, R., Frühling, U., Budzyn, F., Wurth, W., Föhlisch, A. \& Drescher, M. (2008). New J. Phys. 10, 033026.

Mancuso, A. P., Aquila, A., Batchelor, L., Bean, R. J., Bielecki, J., Borchers, G., Doerner, K., Giewekemeyer, K., Graceffa, R., Kelsey, O. D., Kim, Y., Kirkwood, H. J., Legrand, A., Letrun, R., Manning, B., Lopez Morillo, L., Messerschmidt, M., Mills, G., Raabe, S., Reimers, N., Round, A., Sato, T., Schulz, J., Takem, C. S., Sikorski, M., Stern, S., Thute, P., Vagovic P. \& Weinhausen B. (2019). J. Synchrotron Rad. 26, 660-676.

Ozkan, C. (2012). European XFEL Technical Report, XFEL.EU TR2012-004. European XFEL Facility, Hamburg, Germany.

Ozkan, C., Freund, W., Rehanek, J., Buck, J., Zizak, I., Grünert, J., Schaefers, F., Erko, A. \& Molodtsov, S. (2012). Proc. SPIE, 8504, 85040X7.

Pellegrini, C., Marinelli, A. \& Reiche, S. (2016). Rev. Mod. Phys. 88, 015006.

Roth, T., Freund, W., Boesenberg, U., Carini, G., Song, S., Lefeuvre, G., Goikhman, A., Fischer, M., Schreck, M., Grünert, J. \& Madsen, A. (2018). J. Synchrotron Rad. 25, 177-188.

Saldin, E. L., Schneidmiller, E. V. \& Yurkov, M. V. (1999). The Physics of Free-Electron Lasers, 1st ed. Berlin: Springer-Verlag.

Sorokin, A. A., Jastrow, U., Bonfigt, S. \& Tiedtke, K. (2011). XGMD Final Design, European XFEL and DESY Internal Report. Alfresco Document Id: 19789. European XFEL/DESY, Hamburg, Germany.

Syresin, E. et al. (2012). European XFEL Report EDMS-D3595161. European XFEL Facility, Hamburg, Germany.

Syresin, E., Brovko, O., Kapishin, M., Shabunov, A., Yurkov, M., Freund, W., Gruenert, J. \& Sinn, H. (2011). Proceedings of the 2nd International Particle Accelerator Conference (IPAC2011), 4-9 September 2011, San Sebastian, Spain.

Syresin, E., Grebentsov, A. Y., Shabunov, A. V., Zamiatin, N. I., Basta, R., Fiala, T., Hedbavny, P., Brovko, O. I., Freund, W., Gruenert, J., Sinn, H., Novikov, D. \& Yurkov, M. (2014). Proceedings of the 5th International Particle Accelerator Conference (IPAC2014), 15-20 June 2015, Dresden, Germany. WEPRO094.

Tanaka, T., Goto, S., Hara, T., Hatsui, T., Ohashi, H., Togawa, K., Yabashi, M. \& Tanaka, H. (2012). Phys. Rev. ST Accel. Beams, 15, 110701.

Tiedtke, K. K., Feldhaus, J., Hahn, U., Jastrow, U., Nunez, T., Tschentscher, T., Bobashev, S. V., Sorokin, A. A., Hastings, J. B., Möller, S., Cibik, L., Gottwald, A., Hoehl, A., Kroth, U., Krumrey, M., Schöppe, H., Ulm, G. \& Richter, M. (2008). J. Appl. Phys. 103, 094511.

Tono, K., Kudo, T., Yabashi, M., Tachibana, T., Feng, Y., Fritz, D., Hastings, J. \& Ishikawa, T. (2011). Rev. Sci. Instrum. 82, 023108.

Tschentscher, T., Bressler, C., Grünert, J., Madsen, A., Mancuso, A. P., Meyer, M., Scherz, A., Sinn, H. \& Zastrau, U. (2017). Appl. Sci. 76, 592 .

Welch, J., Brachmann, A., Decker, F.-J., Ding, Y., Emma, P., Fisher, A., Frisch, J., Huang, Z., Iverson, R., Loos, H., Nuhn, H.-D., Stefan, P., Ratner, D., Turner, J., Wu, J., Xiang, D., Bionta, R. \& Sinn, H. (2009). Proceedings of the 31st International Free Electron Laser Conference (FEL2009), 23-28 Aug 2009, Liverpool, UK. THOA05. Zhu, D., Cammarata, M., Feldkamp, J. M., Fritz, D. M., Hastings, J. B., Lee, S., Lemke, H. T., Robert, A., Turner, J. L. \& Feng, Y. (2012). Appl. Phys. Lett. 101, 034103. 